\title{
Tissue regeneration observed in a porous acellular bovine pericardium used to repair a myocardial defect in the right ventricle of a rat model
}

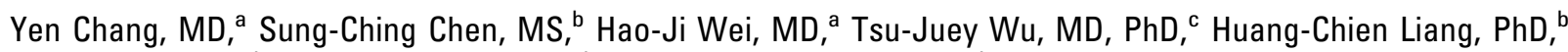
Po-Hong Lai, MS, ${ }^{\text {b }}$ Hang-Hsing Yang, $\mathrm{BS}^{\text {b }}{ }^{\text {and }}$ ansing-Wen Sung, $\mathrm{PhD}^{\mathrm{b}}$

Additional figures are available online. $\widetilde{\theta}$
From the Division of Cardiovascular Surgery, Veterans General Hospital-Taichung, and the College of Medicine, National Yang-Ming University, ${ }^{a}$ Taipei, Taiwan, Republic of China; the Department of Chemical Engineering, National Tsing Hua University, ${ }^{\mathrm{b}}$ Hsinchu, Taiwan, Republic of China; and the Division of Cardiology, Veterans General Hospital-Taichung, and the College of Medicine, National Yang-Ming University, ${ }^{\mathrm{c}}$ Taipei, Taiwan, Republic of China.

Supported by grants from the Ministry of Economic Affair, Taiwan, Republic of China (91-EC-17A-17S1-0009); the Veterans General Hospital, Tsing-Hua, YangMing Joint Research Program (VTY-91P4-23); and the National Health Research Institute (NHRI-EX92-9221EI).

Received for publication Oct 24, 2004; revisions received Feb 15, 2005; accepted for publication April 12, 2005.

Address for reprints: Hsing-Wen Sung, $\mathrm{PhD}$, Department of Chemical Engineering/ Biological Engineering Center, National Tsing Hua University, Hsinchu, Taiwan 30013 (E-mail: hwsung @che.nthu.edu.tw).

J Thorac Cardiovasc Surg 2005;130:705-11

$0022-5223 / \$ 30.00$

Copyright (c) 2005 by The American Association for Thoracic Surgery

doi:10.1016/j.jtcvs.2005.04.007
Objective: Nonliving synthetic materials have been widely used to repair myocardial defects; however, material-related failures do occur. To overcome these problems, an acellular bovine pericardium with a porous structure fixed with genipin (the AGP patch) was developed.

Methods: The AGP patch was used to repair a surgically created myocardial defect in the right ventricle of a rat model. A commercially available expanded polytetrafluoroethylene (e-PTFE) patch was used as a control. At retrieval, a computerized mapping system was used to acquire local epicardial electrograms of each implanted sample, and the appearance of each retrieved sample was grossly examined. The retrieved samples were then processed for histologic examination.

Results: The amplitude of local electrograms on the AGP patch increased significantly with increasing implantation duration, whereas only low-amplitude electrograms were observed on the e-PTFE patch throughout the entire course of the study. No aneurysmal dilation of the implanted patches was seen for either studied group. Additionally, no tissue adhesion was observed on the outer (epicardial) surface of the AGP patch, whereas a moderate tissue adhesion was observed on the e-PTFE patch. On the inner (endocardial) surface, intimal thickening was observed for both studied groups; however, no thrombus formation was found. Intact layers of endothelial and mesothelial cells were identified on the inner and outer surfaces of the AGP patch, respectively. At 4 weeks postoperatively, smooth muscle cells, together with neomuscle fibers (with a few neocollagen fibrils), neoglycosaminoglycans, and neocapillaries, were observed to fill the pores in the AGP patch, an indication of tissue regeneration. These observations were more pronounced at 12 weeks postoperatively. In contrast, no apparent tissue regeneration was observed in the e-PTFE patch.

Conclusion: The present study indicated that the AGP patch holds promise to become a suitable patch for surgical repair of myocardial defects.

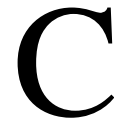
linically, human myocardium lacks the possibility of regeneration. This results in a progressive loss of functional myocardium and a successive reduction in cardiac performance. ${ }^{1} \mathrm{~A}$ patch is often mandatory to repair failing myocardium or congenital cardiac malformations. ${ }^{2}$ Currently, the patches used clinically are made of Dacron polyester fabric, polytetrafluoroethylene (PTFE), glutaraldehyde-treated bovine pericardium, or antibiotic-preserved or cryopreserved homografts. ${ }^{3}$ However, the long-term results have been compromised by materialrelated failures because these materials are not viable, do not grow, and do not provide pulsate flow. ${ }^{3}$

Courtman and colleagues ${ }^{4}$ developed a cell-extraction process to render bovine pericardia free of cells. They hypothesized that cell extraction might remove cellular 
antigens in biologic tissues. Additionally, acellular biologic tissues have been proposed to be used as natural biomaterials for tissue repair and tissue engineering. ${ }^{4,5}$ Natural biomaterials are composed of extracellular matrix proteins that are conserved among different species and that can serve as scaffolds for cell attachment, migration, and proliferation. ${ }^{5}$ This can be a large advantage over synthetic materials.

Our previous study found that the acellular bovine pericardial tissue fixed with genipin can provide a natural microenvironment for host cell migration and might be used as a tissue-engineering extracellular matrix to accelerate tissue regeneration. ${ }^{5}$ Genipin, a naturally occurring cross-linking agent, can be obtained from its parent compound, geniposide, which can be isolated from the fruits of Gardenia jasminoides Ellis. ${ }^{6}$ It has been used to fix biologic tissues or amino groups containing biomaterials for biomedical applications. ${ }^{6}$ It was found that genipin is significantly less cytotoxic than glutaraldehyde. ${ }^{7,8}$ In this study an acellular bovine pericardium with a porous structure fixed by genipin was prepared and used to repair a surgically created myocardial defect in the right ventricle of a rat model.

\section{Materials and Methods}

\section{Preparation of Test Samples}

The procedures used to remove the cellular components from bovine pericardia were based on a method previously reported by Courtman and colleagues ${ }^{4}$ with slight modifications. To increase the pore size and porosity within test samples, the acellular tissues were treated additionally with acetic acid and subsequently with collagenase. ${ }^{9,10}$ Finally, the acellular tissues with a porous structure were fixed in a $0.05 \%$ genipin (Challenge Bioproducts, Taiwan) aqueous solution (phosphate-buffered saline, $\mathrm{pH} 7.4$ ) at $37^{\circ} \mathrm{C}$ for 3 days.

The degree of cross-linking of the genipin-fixed porous acellular bovine pericardium (the AGP patch) was determined by measuring its fixation index and denaturation temperature $(\mathrm{n}=$ 5). ${ }^{11}$ The fixation index, determined by the ninhydrin assay, was defined as the percentage of free amino groups in test tissues reacted with genipin subsequent to fixation. After preparation of test samples, the AGP patch was processed for light microscopic and scanning electron microscopic examinations to investigate its ultrastructures. ${ }^{12}$ The pore size of the AGP patch, stained with hematoxylin and eosin (H\&E), was determined with a microscope. The porosity of the AGP patch was measured by helium pycnometery. ${ }^{13}$ Mechanical testing of the AGP patch was conducted by an Instron material testing machine (Mini 44, Canton, Mass). ${ }^{14}$

The prepared AGP patch was sterilized in a graded series of ethanol solutions for the animal study. A commercially available expanded PTFE (e-PTFE) patch (Gore-Tex patch; W. L. Gore \& Associates, Inc, Flagstaff, Ariz) was used as a control.

\section{Animal Study}

Animal care and use was performed in compliance with the "Guide for the Care and Use of Laboratory Animals" prepared by the Institute of Laboratory Animal Resources, National Research
Council, and published by the National Academy Press, revised 1996. The studied patches $(7.0 \times 7.0 \mathrm{~mm})$ were used to repair transmural defects surgically created in the right ventricles of rat hearts (male S. D. rats, National Laboratory Animal Centers, Taiwan; weight, $400-450 \mathrm{~g}$ ) on the basis of a method reported by Ozawa and associates. ${ }^{15}$ The implanted samples were retrieved at 4 and 12 weeks postoperatively $(n=5$ rats for the AGP patch and $\mathrm{n}=3$ rats for the e-PTFE patch at each time point) and were used for histologic examination.

\section{Computerized Mappings (Epicardial Electrograms)}

In this study a CardioMap mapping system with a plaque electrode array (Prucka Engineering, Houston, Tex) was used to acquire the epicardial electrograms of the implanted patch and its adjacent rat native myocardium immediately after implantation and at retrieval. The plaque electrode array consisted of 112 bipolar electrodes (a 7 $\times 16$ array spanning across a $2.2 \times 6.0-\mathrm{cm}$ rectangular surface) with an interelectrode distance of $3.0 \mathrm{~mm} .{ }^{16}$ The implanted samples were then retrieved, and the appearance of each retrieved sample was grossly examined and photographed.

\section{Histologic Examinations}

The samples used for light microscopy were fixed in $10 \%$ phosphate-buffered formalin and prepared for histologic examination. In the histologic examination the fixed samples were embedded in paraffin, sectioned to a thickness of $5 \mu \mathrm{m}$, and then stained with H\&E. Also, sections of test samples were stained with Masson trichrome and elastic van Gieson (EVG) for the detection of collagen fibrils and muscle fibers and stained with safranin-O to visualize glycosaminoglycans. Additional sections were stained with a van Gieson solution to visualize mesothelial cells. ${ }^{17}$

Immunohistologic staining of smooth muscle cells was performed on deparaffinized sections with a monoclonal antibody against $\alpha$-smooth muscle actin ( $\alpha$-SMA; DAKO Corp, Carpinteria, Calif) and revealed by a peroxidase-antiperoxidase technique. ${ }^{18}$ Five different microscopic fields $(400 \times$ by ECLIPSE-E800; Nikon, Tokyo, Japan) of each patch portion of the right ventricular wall were randomly selected. The area of each studied patch staining positively for $\alpha$-SMA per microscopic field area was counted with a computer-based image-analysis system (Image-Pro Plus; Media Cybernetics, Silver Spring, Md) and converted to percentages. ${ }^{15,19}$ Additionally, the depth of cells positively stained with a monoclonal antibody against $\alpha$-SMA ( $\alpha$-SMA-positive cells) infiltrated into each studied patch was quantified with the same image-analysis system (as a percentage of the depth of the whole test sample). ${ }^{19}$ Additional sections were stained for factor VIII with immunohistologic technique with a monoclonal antifactor VIII antibody (DAKO). ${ }^{20}$

\section{Results}

\section{Test Samples}

The bovine pericardium before cell extraction showed a number of intact cells embedded within the connective tissue matrix (Figure E1 a), whereas the acellular bovine pericardium treated with acetic acid revealed large open spaces (pores; Figure E1, $c$ ). After additional treatment with collagenase (Figure E1, $b$ and $d$ ), the pores in the acellular 
bovine pericardium (the AGP patch) appeared larger, with increased interconnectivity. The pore size and porosity of the AGP patch were $159.8 \pm 26.7 \mu \mathrm{m}$ and $94.9 \% \pm 1.7 \%$, respectively, whereas its denaturation temperature and fixation index were $75.5^{\circ} \mathrm{C} \pm 0.3^{\circ} \mathrm{C}$ and $57.8 \% \pm 5.4 \%$, respectively $(\mathrm{n}=5)$. The ultimate tensile strength of the AGP patch was $2.1 \pm 0.3 \mathrm{MPa}(\mathrm{n}=5)$.

\section{Computerized Mappings (Epicardial Electrograms)}

Immediately after implantation, low-amplitude electrogram signals were observed on both the e-PTFE and AGP patches (Figure 1, $b$ and $h$ ). At 4 and 12 weeks postoperatively, the mappings of the epicardial electrograms on the e-PTFE patch still showed low amplitudes compared with those observed on its adjacent native myocardium (Figure 1, $d$ and $f$ ). In contrast, the amplitude of the local electrograms on the epicardial surface of the AGP patch increased significantly with increasing implantation duration (Figure $1, j$ and $l$ ).

\section{Gross Examination}

At retrieval, on the outer surface, no apparent tissue adhesion was observed on the AGP patch for all the animals studied, whereas a moderate adhesion to the chest wall was seen for the e-PTFE patch in one of the animals studied (n $=3$ at each time point). On the inner surface, intimal thickening was observed for both studied groups; however, no thrombus formation was found. No aneurysmal dilation of the implanted patches was seen for either studied group throughout the entire course of the study.

\section{Histologic Findings}

At 4 weeks postoperatively, intimal thickening was observed on the inner surfaces of both studied groups (Figure $\mathrm{E} 2, a$ and $b$ ). Host cells, together with neotissue fibrils and neocapillaries, were clearly observed in the inner and outer layers of the AGP patch (Figure e2, $b$ and $d$ ). In contrast, only a few infiltrated cells (mostly inflammatory cells) were found in the innermost and outermost layers of the e-PTFE patch (Figure e2, $a$ and $c$ ). Endothelial-like cells were visible on the inner surfaces of both studied groups (Figure e2, $a$ and $b$ ). Additionally, mesothelial-like cells were observed on the outer surface of the AGP patch (Figure E2, $d$ ). However, no mesothelial-like cells were found on the outer surface of the e-PTFE patch (Figure E2, $c$ ). Instead, fibrous tissue was firmly attached to the outer surface of the e-PTFE patch.

Degradation of the structural fibrils (collagen network) in the AGP patch was observed. Additionally, host cells, together with neotissue fibrils, were found to fill some of the pores in the middle layer of the AGP patch (Figure E3, $b$ ). In contrast, no apparent degradation was observed for the e-PTFE patch, and neither cells nor neotissue fibrils were observed in the e-PTFE patch (Figure E3, $a$ ). The neotissue fibrils regenerated in the AGP patch were identified to be neomuscle fibers (stained red) with a few neocollagen fibrils (stained blue), as determined with Masson trichrome stain (Figure E3, $d$ ). The neomuscle fibers seen in the AGP patch were further confirmed with EVG stain (stained brown, Figure E3, $f$ ). Also, there were some neoglycosaminoglycans regenerated within the pores of the AGP patch recognized by safranin-O stain (stained pink; Figure E3, $h$ ).

An intact layer of endothelial cells was identified on the intimal thickening generated on the inner (endocardial) surface of the AGP patch by the factor VIII staining (Figure 2, $B)$. In contrast, endothelial cells did not universally and totally cover the entire inner surface of the e-PTFE patch (Figure 2,a). The outer surface of the AGP patch was positively stained with van Gieson stain (Figure 2,d), indicating the presence of mesothelial cells. However, no mesothelial cells were observed on the outer surface of the e-PTFE patch (Figure 2,c). Additionally, $\alpha$-SMA positively stained cells were observed in the middle layer of the AGP patch (Figure 2, $f$ ).

At 12 weeks postoperatively, there were still no signs of tissue regeneration (host cells and neotissue fibrils) observed inside the e-PTFE patch (Figure 3, $a, c$, and $e$ ). Compared with those observed at 4 weeks postoperatively, a more apparent degradation in the structural fibrils of the AGP patch was found (Figure 3,b), whereas the neomuscle fibers seen were more compact and organized (Figure 3,d). The area of cells positively stained with a monoclonal antibody against $\alpha$-SMA in the AGP patch $(24.5 \% \pm 3.2 \%$ of the entire patch; Figure 3, $f$ ) appeared to be larger than that observed at 4 weeks postoperatively $(9.0 \% \pm 2.0 \%$; Figure 2, $f$ ). Additionally, the depth of the $\alpha$-SMA-positive cells infiltrated into the AGP patch $(98.7 \% \pm 2.6 \%)$ was significantly greater than its counterpart found at 4 weeks postoperatively $(57.8 \% \pm 7.9 \%)$.

\section{Discussion}

We found that the e-PTFE and AGP patches did not thin and dilate as a patch to repair a myocardial defect created in the right ventricle of a rat model throughout the entire course of the study. This indicated that the mechanical strengths of both studied groups were strong enough to tolerate the right ventricular pressure without aneurysmal dilation during the long-term implantation. The e-PTFE patch has been accepted worldwide as one of the most reliable nondegradable synthetic materials in terms of durability, low thrombogenicity, and comfortable handling for surgical intervention. ${ }^{21}$ Additionally, an advantage of the e-PTFE patch is that it does not thin and dilate in response to intraventricular pressure. ${ }^{15}$ Unfortunately, the e-PTFE patch is not viable, does not provide pulsatile flow, and does not grow as a child's heart or blood vessel grows. ${ }^{22}$ 


\section{e-PTFE}

(a)

(b)
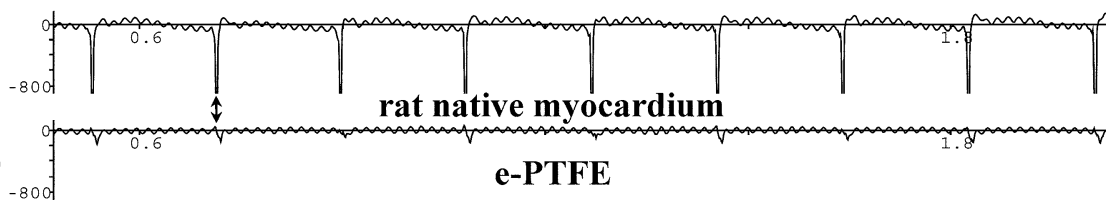

(c)

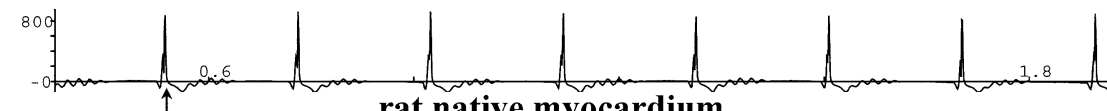

(d)

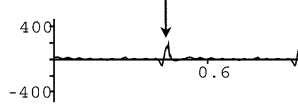

rat native myocardium

(e)

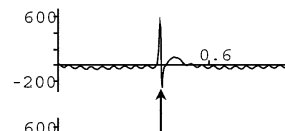

(f)

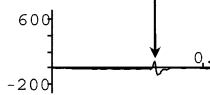

e-PTFE

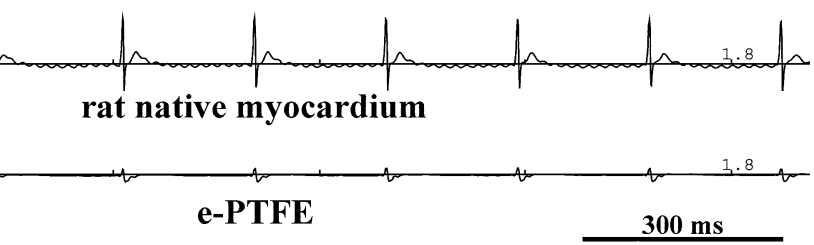

\section{AGP}

(g)

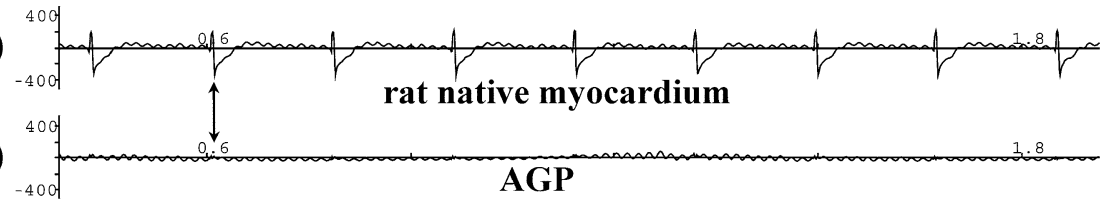

(h)

(i)

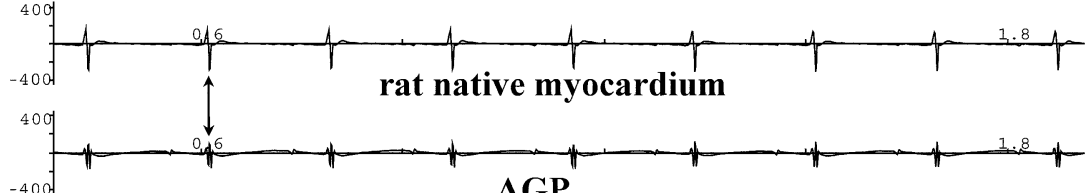

(k)

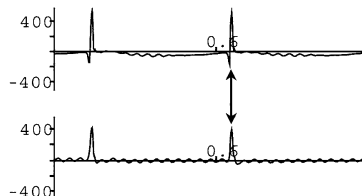

AGP

rat native myocardium

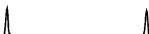

AGP

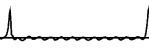

1 A.

$300 \mathrm{~ms}$

Figure 1. Local electrograms obtained from the electrodes located on the epicardial surfaces of the e-PTFE and AGP patches and their adjacent rat native myocardia immediately after implantation and those observed at 4 and 12 weeks postoperatively. $a$ and $b$, e-PTFE immediately after implantation; $c$ and d, e-PTFE at 4 weeks postoperatively; $e$ and $f, e$-PTFE at 12 weeks postoperatively; $g$ and $h, A G P$ immediately after implantation; $i$ and $j, A G P$ at 4 weeks postoperatively; $k$ and $\mathrm{I}, \mathrm{AGP}$ at 12 weeks postoperatively.
As indicted in Figure E2, $d$, the outer (epicardial) layer of the AGP patch retrieved at 4 weeks postoperatively became well integrated with the host tissue, as shown by our histologic examination. (Host cells, together with neotissue fibrils and neocapillaries, were noted in the outer layer of the AGP patch.) Additionally, there was an intact layer of neomesothelial cells, identified by using the van Gieson stain, observed on the outer surface of the AGP patch (Figure 2, d). However, such observations did not occur in or on the e-PTFE patch (Figure E2, c, and Figure 2,c). Instead, fibrous tissue was firmly attached to the outer surface of the e-PTFE patch. It is known that the epicardium forms the outer covering of the heart and has an external layer of flat mesothelial cells. ${ }^{23}$ It is well documented that mesothelial cells prevent adhesions. ${ }^{24}$ Whitaker and coworkers $^{24}$ reported that a pure culture of mesothelial cells was able to induce fibrinolysis. These results likely explain the observation that once the surface of the AGP patch was populated with mesothelial cells, it remained resistant to adhesion formation.

At 4 weeks postoperatively, intimal thickening covered with endothelial cells was found on the inner (endocardial) surfaces of the e-PTFE and AGP patches. This finding suggested that host endocardial endothelial cells or endothelial progenitor cells were involved in the endothelialization on the inner surfaces of the implanted patches. ${ }^{3,25}$ No 


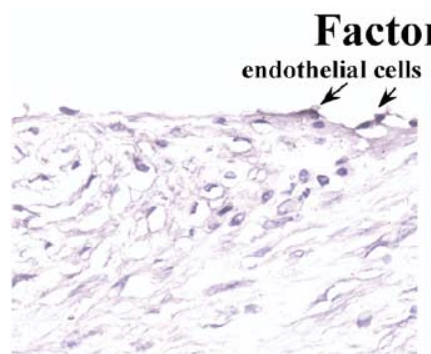

(a) e-PTFE, 4-Week Postop.

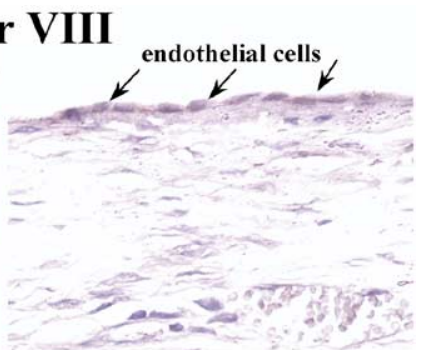

(b) AGP, 4-Week Postop.

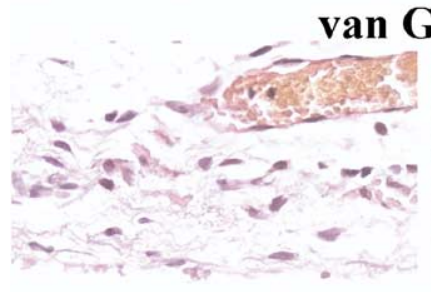

(c) e-PTFE, 4-Week Postop.

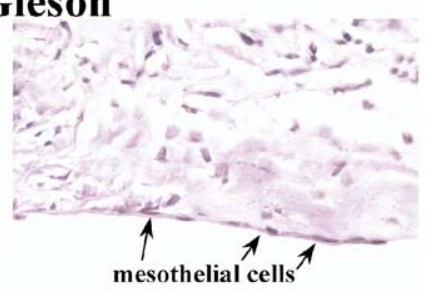

(d) AGP, 4-Week Postop. $\alpha$-Smooth Muscle Actin

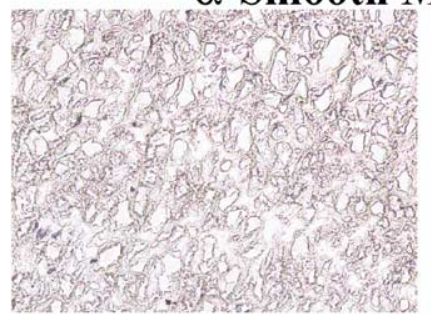

(e) e-PTFE, 4-Week Postop.

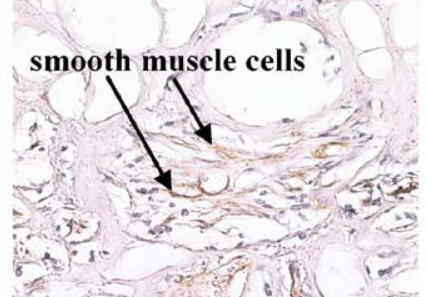

(f) AGP, 4-Week Postop.
Figure 2. Photomicrographs of the e-PTFE and AGP patches stained with an antibody against factor VIII (a and $b$ : original magnification, $800 \times$ ), van Gieson (c and d: original magnification, $800 \times$ ), and a monoclonal antibody against $\alpha$-SMA (e and f: original magnification, $400 \times$ ) retrieved at 4 weeks postoperatively.

thrombus formation was observed in either studied sample. Similar results were also observed in our previous study in using the genipin-fixed acellular bovine pericardium as a patch to repair a defect created in the pulmonary trunk in a canine model. ${ }^{26}$

As indicated by the H\&E, Masson trichrome, EVG, and safranin-O stains (Figure E3, $b, d, f$, and $h$ ), host cells, together with neomuscle fibers (with a few neocollagen fibrils), neoglycosaminoglycans, and neocapillaries, were observed to fill the pores in the AGP patch at 4 weeks postoperatively, an indication of tissue regeneration. Additionally, the middle layer of the AGP patch was positively stained with a monoclonal antibody against $\alpha$-SMA (Figure $2, f$, and Figure $3, f)$, indicating that myofibroblasts or smooth muscle cells were observed in the AGP patch. It is known that myofibroblasts show an immunohistochemical expression of $\alpha$-SMA. ${ }^{27}$ Collagen synthesis might be one of
H\&E

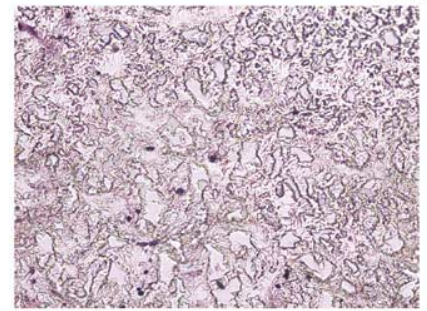

(a) e-PTFE, 12-Week Postop. Masson's 'Tr

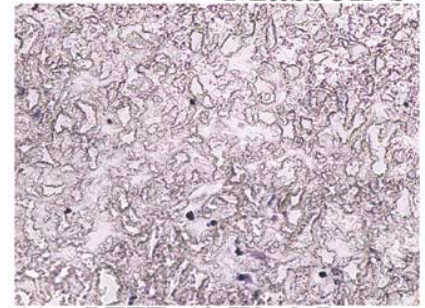

(c) e-PTFE, 12-Week Postop. $\alpha$-Smooth Muscle Actin

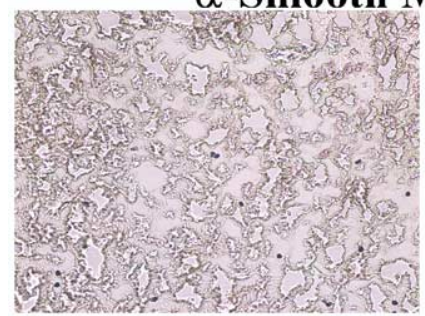

(e) e-PTFE, 12-Week Postop.

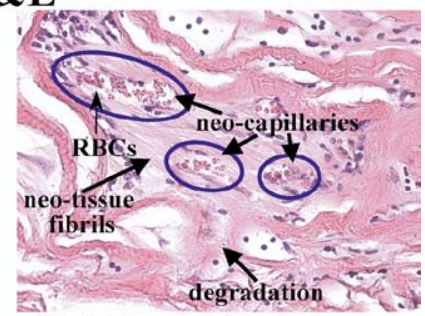

(b) AGP, 12-Week Postop.

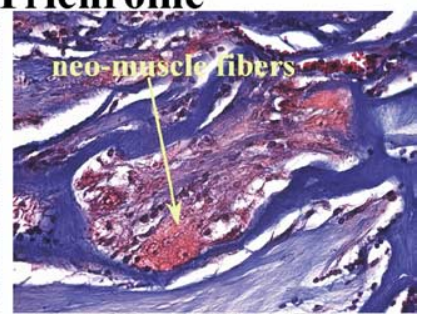

(d) AGP, 12-Week Postop.

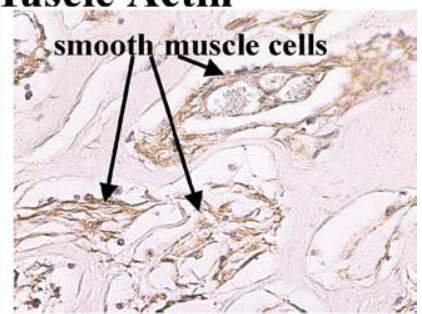

(f) AGP, 12-Week Postop.

Figure 3. Photomicrographs of the middle layers of the e-PTFE and AGP patches stained with H\&E ( $a$ and $b)$, Masson trichrome (c and d), and a monoclonal antibody against $\alpha$-SMA (e and f) retrieved at 12 weeks postoperatively. (Original magnification, $400 \times$.)

the important roles of myofibroblasts, supplementing the damaged area where the parenchymal tissue is defective (scar formation). ${ }^{27,28}$

On the other hand, smooth muscle cells permit formation of a muscular tissue (observed in our AGP patch: stained red with Masson trichrome, Figure E3, $d$, and Figure 3, $d$; stained brown with EVG, Figure E3, $f$ ) in addition to collagen formation (stained blue with Masson trichrome). ${ }^{29}$ Therefore, the $\alpha$-SMA-positive cells observed in the AGP patch appeared to be smooth muscle cells (Figure 2, $f$, and Figure $3, f$ ) rather than myofibroblasts. Accordingly, migration of smooth muscle cells into the AGP patch occurred and resulted in artificial muscle-like tissues. ${ }^{30}$ This finding suggested that host progenitor cells from the systemic circulation or from the surrounding tissue might be relevant to the presence of smooth muscle cells in the AGP patch. ${ }^{25}$ The aforementioned observations were more pronounced at 12 weeks postoperatively (Figure $3, b, d$, and $f$ ). 
Ozawa and associates ${ }^{15}$ reported that the smooth muscle cell-seeded biodegradable patches can be used to repair the right ventricular outflow tract. The seeded smooth muscle cells survived in scaffolds for 8 weeks and led to new muscular formation. In their study the smooth muscle cells were chosen because they can be harvested from a number of sources in the donor and returned to the donor as an autologous cell patch. It was reported that smooth muscle cells can respond to in vivo mechanical stress by hyperplasia and hypertrophy to prevent patch dilation and thinning and improve myocardial function. ${ }^{30}$ Additionally, it is known that smooth muscle cells are one of the specialized contractile cell types distinguished in the human body. ${ }^{31} \mathrm{An}$ autologous, cell-seeded, biodegradable patch might preserve the structure of the ventricular wall while providing the potential for growth and contractility.

In contrast, only a few infiltrated inflammatory cells were present in the most outer and inner layers of the e-PTFE patch (Figure E2, $a$ and $c$ ). Also, no apparent tissue regeneration was observed in the middle layer of the e-PTFE patch (Figure E3, $a, c, e$, and $g$, and Figure 3, $a, c$, and $e$ ), whereas only low-amplitude electrogram signals were observed on the epicardial surface of the e-PTFE patch throughout the entire course of the study (Figure 1, $d$ and $f$ ). On the contrary, the amplitude of the local electrograms on the AGP patch increased significantly with increasing implantation duration (Figure $1, j$ and $l$ ), an indication of better electrical conductance. The increase in the amplitude of the local electrograms on the AGP patch might be due to the regenerated tissues observed in its pores.

In this study, smooth muscle cells, together with neomuscle fibers, were observed in the AGP patch, an indication of tissue regeneration. However, cardiomyocytes were not found within the AGP patch, a significant limitation of this study. In a future study, we plan to seed bone marrow mesenchymal stem cells onto the AGP patch. It was reported that autologous bone marrow cells transplanted into ventricular scar tissue might differentiate into cardiomyocytes and restore myocardial function. ${ }^{32}$ The main benefits of the cardiomyocytes are thought to be an increase in myocardial wall tension and elasticity, which minimize ventricular dilation. ${ }^{33}$

\section{Conclusions}

In conclusion, the AGP patch might preserve the structure of the right ventricle and prevent aneurysmal dilation while providing the potential for tissue regeneration. These results indicated that the AGP patch holds promise to become a suitable patch for surgical repair of myocardial defects.

\section{References}

1. Jugdutt BI. Prevention of ventricular remodelling post myocardial infarction: timing and duration of therapy. Can J Cardiol. 1993;9:10314.
2. Akhyari P, Fedak PWM, Weisel RD, Lee TYJ, Verma S, Mickle DAG, et al. Mechanical stretch regimen enhances the formation of bioengineered autologous cardiac muscle grafts. Circulation. 2002:106(suppl I):I137-42.

3. Ozawa T, Mickle DAG, Weisel RD, Koyama N, Wong H, Ozawa S, et al. Histologic changes of nonbiodegradable and biodegradable biomaterials used to repair right ventricular heart defects in rats. J Thorac Cardiovasc Surg. 2002;124:1157-64.

4. Courtman DW, Pereira CA, Kashef V, MicComb D. Development of a pericardial acellular matrix biomaterial: biochemical and mechanical effects of cell extraction. J Biomed Mater Res. 1994;28:655-66.

5. Chang Y, Tsai CC, Liang HC, Sung HW. In vivo evaluation of cellular and acellular bovine pericardia fixed with a naturally occurring crosslinking agent (genipin). Biomaterials. 2002;23:2447-57.

6. Sung HW, Chen CN, Huang RN, Hsu JC, Chang WH. In vitro surface characterization of a biological patch fixed with a naturally occurring crosslinking agent. Biomaterials. 2000;21:1353-62.

7. Sung HW, Huang RN, Huang LLH, Tsai CC. In vitro evaluation of cytotoxicity of a naturally occurring cross-linking reagent for biological tissue fixation. J Biomater Sci Polym Ed. 1999;10:63-78.

8. Chang Y, Tsai CC, Liang HC, Sung HW. Reconstruction of the right ventricular outflow tract with a bovine jugular vein graft fixed with a naturally occurring crosslinking agent (genipin) in a canine model. J Thorac Cardiovasc Surg. 2001;122:1208-18.

9. Chang Y, Lee MH, Liang HC, Hsu CK, Sung HW. Acellular bovine pericardia with distinct porous structures fixed with genipin as an extracellular matrix. Tissue Eng. 2004;10:881-92.

10. Wei HJ, Liang HC, Lee MH, Huang YC, Chang Y, Sung HW. Construction of varying porous structures in acellular bovine pericardia as a tissue-engineering extracellular matrix. Biomaterials. 2005; 26:1905-13.

11. Sung HW, Chang Y, Chiu CT, Chen CN, Liang HC. Crosslinking characteristics and mechanical properties of a bovine pericardium fixed with a naturally occurring crosslinking agent. J Biomed Mater Res. 1999;47:116-26.

12. Ramshaw AM, Casagranda F, White JF, Edwards GA, Hunt JA, Williams DF, et al. Effects of mesh modification on the structure of a mandrel-grown biosynthetic vascular prosthesis. J Biomed Mater Res. 1999;47:309-15.

13. Martin I, Shastri VP, Padera RF, Yang J, Mackay AJ, Langer R, et al. Selective differentiation of mammalian bone marrow stromal cells cultured on three-dimensional polymer foams. J Biomed Mater Res. 2001;55:229-35.

14. Lee JM, Haberer SA, Boughner DR. The bovine pericardial xenograft. I: effect of fixation in aldehydes without constraint on the tensile viscoelastic properties of bovine pericardium. J Biomed Mater Res. 1989;23:457-75.

15. Ozawa T, Mickle DAG, Weisel RD, Koyama N, Ozawa S, Li RK. Optimal biomaterial for creation of autologous cardiac grafts. Circulation. 2002:106(suppl I):I176-82.

16. Wu TJ, Doshi RN, Huang HLA, Blanche C, Kass RM, Trento A, et al. Simultaneous biatrial computerized mapping during permanent atrial fibrillation in patients with organic heart disease. J Cardiovasc Electrophysiol. 2002;13:571-7.

17. Prophet EB, Mills B, Arrington JB, Sobin LH. Laboratory methods in histotechnology. 2nd ed. Washington: American Registry of Pathology; 1994. p. 136.

18. Allaire E, Guettier C, Bruneval P, Plissonnier D, Michel JB. Cell-free arterial grafts: Morphologic characteristics of aortic isografts, allografts, and xenografts in rats. J Vasc Surg. 1994;19:446-56.

19. Courtman DW, Errett BF, Wilson GJ. The role of crosslinking in modification of the immune response elicited against xenogenic vascular acellular matrices. J Biomed Mater Res. 2001;55:576-86.

20. Bader A, Schiling T, Teebken OE. Tissue engineering of heart valveshuman endothelial cell seeding of detergent acellularized porcine valves. Eur J Cardiothorac Surg. 1998;14:279-84.

21. Izutani H, Gundry SR, Vricella LA, Xu H, Bailey LL. Right ventricular outflow tract reconstruction using a Gore-Tex membrane monocusp valve in infant animals. ASAIO J. 2000;46:553-5. 
22. Uemura H, Yagihara T, Kawahira Y, Yoshikawa Y, Kitamura S. Total cavopulmonary connection in children with body weight less than 10 kg. Eur J Cardiothorac Surg. 2000;17:543-9.

23. Macchi E, Cavalieri M, Stilli D, Musso E, Baruffi S, Olivetti G, et al. High-density epicardial mapping during current injection and ventricular activation in rat hearts. Am J Physiol Heart Circ Physiol. 1998; 275:H1886-97.

24. Whitaker D, Papadimitriou JM, Walters M. The mesothelium: its fibrinolytic properties. J Pathol. 1982;136:291-9.

25. Scott SM, Barth MG, Gaddy LR, Ahl ET Jr. The role of circulating cells in the healing of vascular prostheses. J Vasc Surg. 1994;19:58593.

26. Chang Y, Liang HC, Wei HJ, Chu CP, Sung HW. Tissue regeneration patterns in acellular bovine pericardia implanted in a canine model as a vascular patch. J Biomed Mater Res. 2004;69A:323-33.

27. Takemura G, Ohno M, Hayakawa Y, Misao J, Kanoh M, Ohno A, et al. Role of apoptosis in the disappearance of infiltrated and proliferated interstitial cells after myocardial infraction. Circ Res. 1998;82:1130-8.
28. Schürch W, Seemayer TA, Gabbiani G. Myofibroblast. In: Sternberg SS, editor. Histology for pathologists. New York: Raven Press Publishers; 1992. p. 109-44.

29. Shinoka T, Ma PX, Shum-Tim D, Breuer CK, Cusick RA, Zund G, et al. Tissue-engineered heart valves: autologous valve leaflet replacement study in a lamb model. Circulation. 1996:94II164-8.

30. Yoo KJ, Li RK, Weisel RD, Mickle DAG, Li G, Yau TM. Autologous smooth muscle cell transplantation improved heart function in dilated cardiomyopathy. Ann Thorac Surg. 2000;70:859-65.

31. Amenta PS, Amenta PS. Muscle tissue. In: Elias H, Pauly JE, editors. Histology from normal microanatomy to pathology. Padova: Piccin Nuova Libraria S.p.A.; 1997. p. 153.

32. Orlic D, Kajstura J, Chimenti S, Jakoniuk I, Anderson SM, Li B, et al. Bone marrow cells regenerate infracted myocardium. Nature. 2001; 410:701-5.

33. Li RK, Jia ZQ, Weisel RD, Mickle DAG, Zhang J, Mohabeer MK, et al. Cardiomyocyte transplantation improved heart function. Ann Thorac Surg. 1996;62:654-61. 


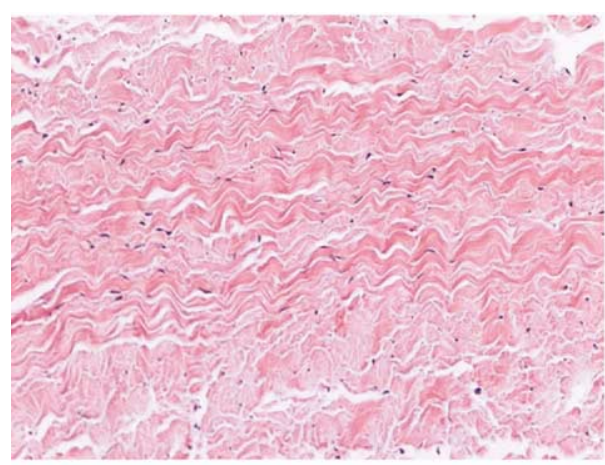

(a)

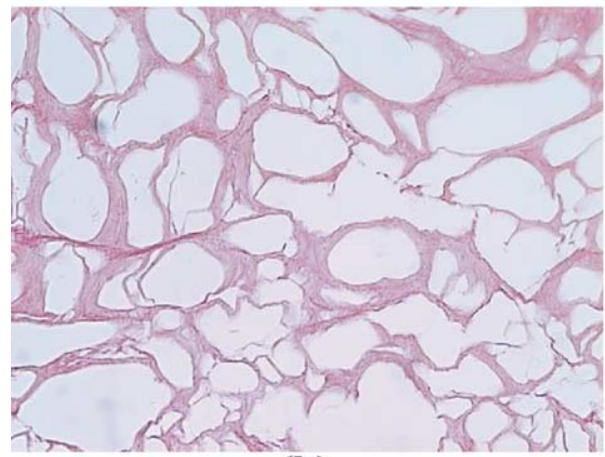

(b)

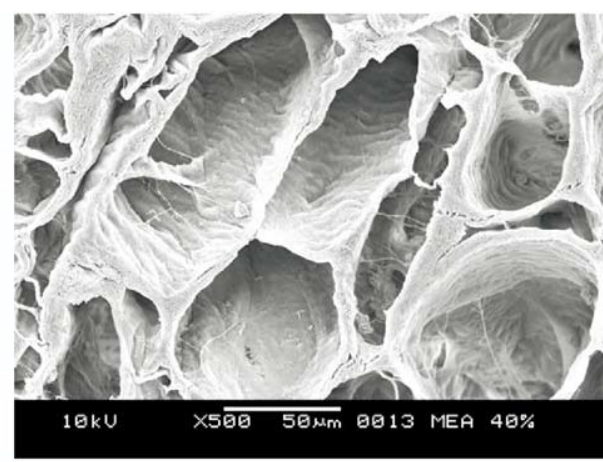

(c)

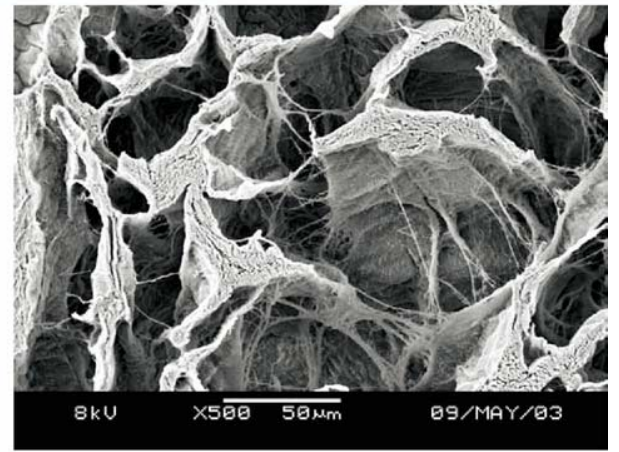

(d)

Figure E1. Photomicrographs of the bovine pericardium before cell extraction (a) and the prepared AGP patch (b), with the acellular bovine pericardium additionally treated with acetic acid, subsequently treated with collagenase, and stained with H\&E (original magnification, $200 \times$ ), and scanning electron microscopic micrographs of the acellular bovine pericardium treated with acetic acid (c) and further treated with collagenase (d; the AGP patch). 

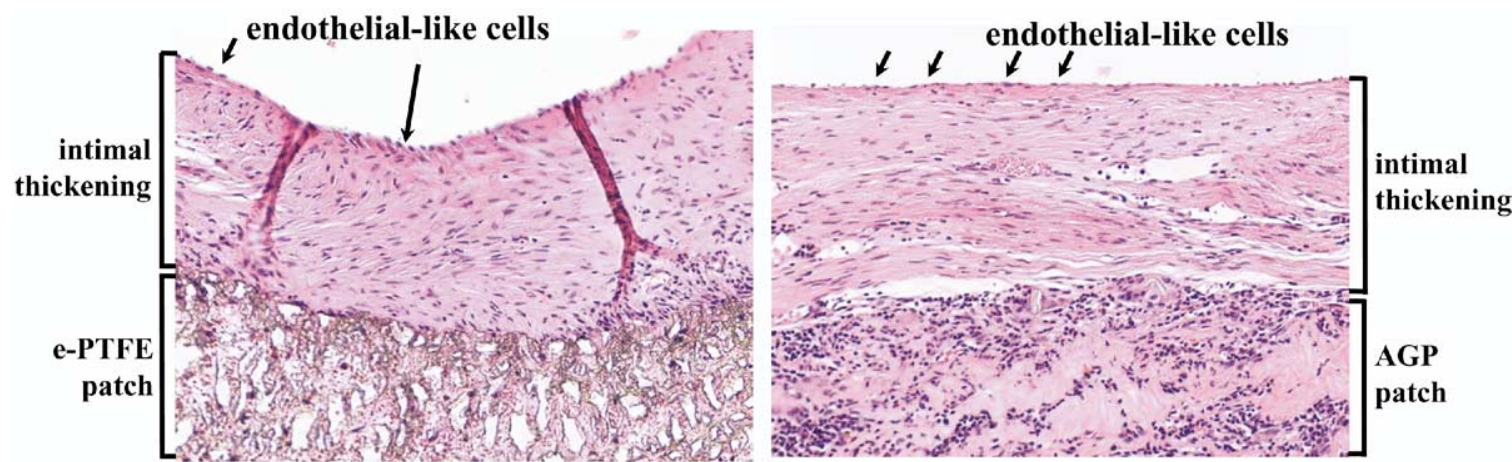

(a)

Inner Layer

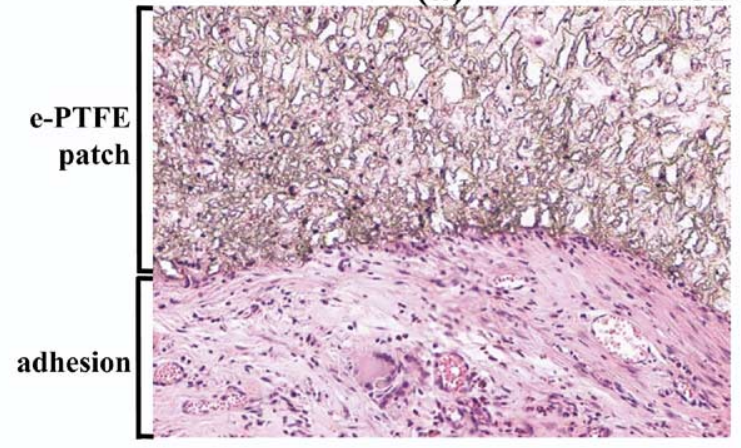

(c)

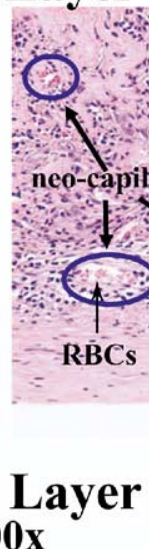

(b) intimal

thickening

Figure E2. Photomicrographs of the inner (endocardial) layers of the e-PTFE patch (a) and the AGP patch (b) and the outer (epicardial) layers of the e-PTFE patch (c) and the AGP patch (d) stained with H\&E retrieved at 4 weeks postoperatively. (Original magnification $200 \times$.) 


\section{H\&E}

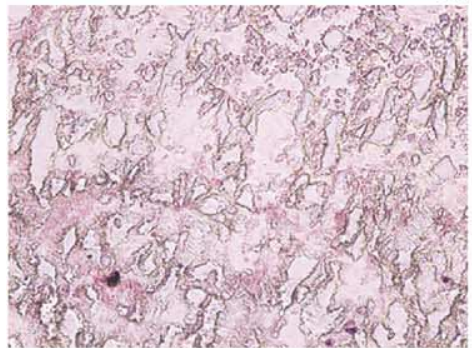

(a) e-PTFE, 4-Week Postop.

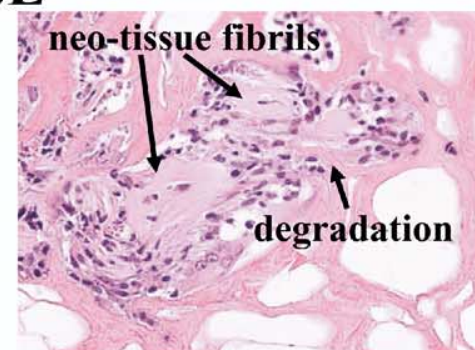

Masson's Trichrome

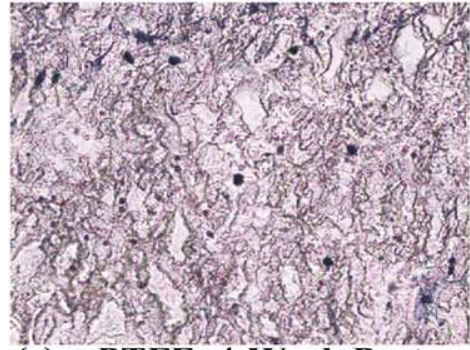

(c) e-PTFE, 4-Week Postop.

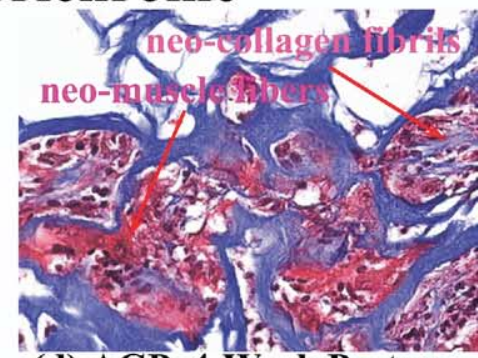

EVG

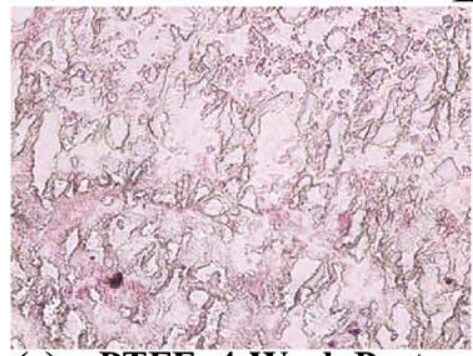

(e) e-PTFE, 4-Week Postop.

(d) AGP, 4-Week Postop.

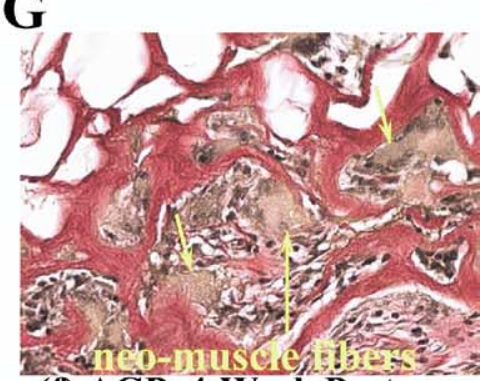

(f) AGP, 4-Week Postop.

\section{Safranin-O}

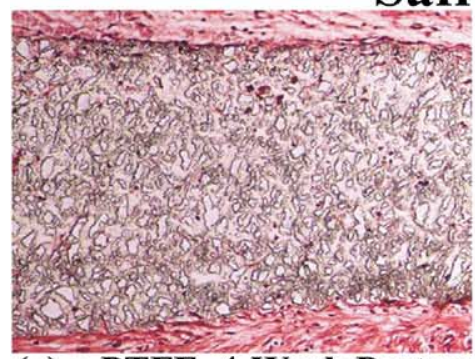

(g) e-PTFE, 4-Week Postop.

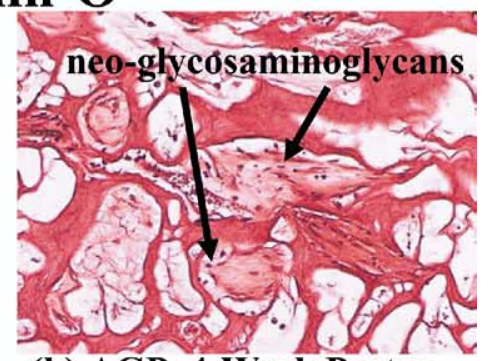

(h) AGP, 4-Week Postop.

Figure E3. Photomicrographs of the middle layers of the e-PTFE and AGP patches stained with H\&E (a and b: original magnification $400 \times$ ), Masson trichrome (c and d: original magnification $400 \times$ ), EVG (e and f: original magnification $400 \times$ ), and safranin- 0 (g and $h$ : original magnification $200 \times$ ) retrieved at 4 weeks postoperatively. 